\title{
Germanica
}

\section{Europa als Labyrinth des Abendlandes}

Michael Köhlmeiers Neu-Lektüre und Neu-Erzählung des Mythos

Europe - The labyrinth of the western world. Michael Köhlmeier's

reinterpretation of the myth

L'Europe, labyrinthe de l'Occident. Relecture et réécriture du mythe chez

Michael Köhlmeier

\section{Maria Behre}

\section{OpenEdition \\ Journals}

Édition électronique

URL : http://journals.openedition.org/germanica/2895

DOI : $10.4000 /$ germanica.2895

ISSN : 2107-0784

\section{Éditeur}

Université de Lille

Édition imprimée

Date de publication : 30 septembre 2015

Pagination : 41-55

ISBN : 9782913857353

ISSN : 0984-2632

\section{Référence électronique}

Maria Behre, «Europa als Labyrinth des Abendlandes », Germanica [Online], 56 | 2015, Online erschienen am: 30 September 2017, abgerufen am 06 Oktober 2020. URL : http://

journals.openedition.org/germanica/2895; DOI : https://doi.org/10.4000/germanica.2895

\section{(c) Tous droits réservés}




\title{
Europa als Labyrinth des Abendlandes \\ Michael Köhlmeiers Neu-Lektüre und Neu-Erzählung des Mythos
}

\author{
Maria BEHRE \\ Einhard-Gymnasium - Aachen
}

\begin{abstract}
I
Am Anfang steht ein dialektisch deutbarer Mythos: Handelt es sich beim Kontakt zwischen der phönizischen Königstochter Europa und Zeus in Stiergestalt um einen Raub mit Vergewaltigung oder um Zuneigung und Liebe, abstrahiert formuliert um eine Urszene der Aggression oder des Sich-Einlassens auf die Freiheit, hier eine Meerfahrt?

Johannes Bobrowski (1917-1965), der Ostberliner Autor aus Tilsit und Königsberg, der sich die historische Schuld der Deutschen gegenüber dem europäischen Osten als Werkthema nach dem Zweiten Weltkrieg wählte, entfaltet diese Dialektik, „die griechische Mythologie als die abendländische Gedächtniskultur"1, allerdings in unveröffentlichten Gedichten des Jahres 1952: Europäische Ode und Europa ${ }^{2}$. Diese Texte vertraute er nur einem Freund aus russischer Kriegsgefangenschaft an. Die Ode baut den Kontrast auf zwischen der Gewaltgeschichte Europas (,Verwesung“, „Verschuldung“, „Mutter du der Menschheit, in deinen blut'gen/ Händen liegt die Kraft der Entsühnung"), die sich in der Shoah
\end{abstract}

1. - Jan Assmann, in Philosophie Magazin, Sonderausgabe 02: „Die griechischen Mythen“, 2014, S. 13.

2. - Für den Hinweis auf Bobrowskis Europa-Gedichte danke ich herzlich Herrn Wilfried Fonrobert, Düsseldorf.

GERMANICA, 2015, LVI, pp. 41 à 55. 
des Zwanzigsten Jahrhunderts als deutsches und europäisches Versagen manifestiert, und einer Gegengeschichte der Befriedung:
$\mathrm{Da}$ in Klagen nicht die Geraubte auffuhr, vielmehr sich erhob und gereckten Halses um sich blickte, mit einer herrlich-leichten Regung der Hand nur
sich des Stiers bemächtigte, der sich sanft schon, willig leiten ließ, seinen Raub behutsam trug sehr weichen Schrittes, das Schnauben seines Atems zurückhielt. ${ }^{3}$

Die leibliche Kraft der Selbstbeherrschung, aus der die Zurückhaltung des Aggressors erwächst, liegt in der StrophenpaarMitte in der Bewegung der Hand, die sich nach den vorausgehenden Versen bisher noch nicht in Malerei visualisieren ließ, wie der studierte Kunstgeschichtler Bobrowski bemerkt, und im Strophenbruch. Nicht Verschweigen, Sich-Recken, Blicken oder Ähnliches, sondern die Handsymbolik bringt Aufregung bzw. Erregung auf eine neue Ebene, die der Macht der Sanftheit, der Behutsamkeit, der Weichheit als Macht gegen jede Gewalttätigkeit.

Im Gedicht Europa wird umgekehrt diese Weichheit als „Legende“ an den Anfang des Gedichts gestellt, in der ersten der drei neunversigen Strophen, um sie in der zweiten historisch zu widerlegen, die ,Verklärung durch das Hören auf das „Ächzen aus dem eingerissenen/ blutigen Mund" aufhebend, formal in der Mitte des Gedichts (V. 14f.). Schließlich werden in der dritten Strophe die „Sänger“ ermutigt, trotz der „Gewalttat" ,jene sanfte Mär“ dem sie anredenden lyrischen „Wir“ (V. 24f., 27) des Publikums weiterhin zu Gehör zu bringen. Die Rezeption dieses Mythos hat nach dem Schluss allerdings die Ebene einer ,unaussöhnlich fernen' Idylle. Mit dem nicht mehr gebräuchlichen Ausdruck für lateinisch ,implacabilis“ in der Nähe zu ,,unversöhnlich, unversöhnbar“ - französisch beides ,conciliant“ - kann auf die Utopie eines Nicht-Mehr bzw. Noch-Nicht hingewiesen werden, die ,Ferne' der Vergangenheit und die ,Ferne' einer Zukunft.

Bobrowski geht aus von der Darstellung der Europa mit dem Stier in der Kunstgeschichte. Dies ist ein Topos, den es zu erneuern gilt, z.

3. - Johannes Bobrowski, Gesammelte Werke in sechs Bänden, hrsg. von Eberhard Haufe, Zweiter Band: Gedichte aus dem Nachlass, Berlin, Union Verlag, 1987, S. 192f., hier S. 193 (Schluss); vgl. das Gedicht Europa, ebd., S. 203f.; vgl. auch Johannes Bobrowski, Spur der Stimmen, hrsg. von Maria Behre, Andreas Degen, Christian Fabritz, Braunschweig, Schroedel Verlag, 2012 und 2013. C. Fabritz danke ich für Support ebenso wie Hedwig Caspers und Golo Kronenwerth. 
B. im Gedicht in Form eines lyrischen Bildes, das bisherige visuelle Nicht-Darstellbarkeit inszeniert, als „Arbeit am Mythos“ nach Hans Blumenberg. Das lyrische Ich fragt sprach-, traditions- und toposkritisch: „Oder soll vergeblich der heil'gen Sage/ zahllose Bedeutung sein, der die Maler nie noch genügten?" 4 Gustav Schwabs Sagen des klassischen Altertums aus den Jahren 1838-1840 ziert in der aktuellen Ausgabe das Motiv ,Europa und der Stier' in zärtlicher, schwebender Umarmung (Patera-Maler um 330 vor Christus), Schwab schildert Europa mit ihrem Traum von den beiden Weltreichen in Frauengestalt, Asien als Heimat und etwas Neues, Fremdes, das ihre Sehnsucht und Gestaltungskraft weckt ${ }^{5}$. Europa sieht den Traum als Vorausdeutung. Sie ist bereit für die Liebe (Rosen im Blumenkörbchen) und begehrt Zeus in himmlischer Gestalt eines zahmen goldgelben Stiers mit silberweißem Stirnmal, sichelmondförmigem Horn und blauen Augen. Zweifel werden zerstreut durch Aphrodites Aufklärung, dass der Stier Zeus sei und Europa als Sterbliche erwählt, Namensgeberin des neuen Erdteils. Den kontrastiven Eckwert verkörpert Max Beckmann, der 1933 ein Gegenbild der Erbeutung eines gefesselt-gefolterten schreienden Opfertiers durch das Stier-Tier schuf.

Die Rezeption des Mythos ist damit bestimmt von der Frage, ob dieser Akt gewaltsam oder freiwillig geschah, als Raub oder als „Verführung zur Freiheit“ (Durs Grünbein, 2003) ${ }^{6}$. Innerhalb der Deutungsgeschichte ist die letztere Deutung modern und mythen- und genderkritisch, denn „Reise“, „Bewegung“, „Seeabenteuer“, „Traum“Sicherheit, Nachgeben gegenüber „Verlockung“, „Vorwitz“ zeugen von einer Dynamik, die autonom bestimmt ist, und die Geschichte ausmachende Lebensbefähigungen wie „Abschied, Umorientierung und Neubeginn" versinnbildlichen und verleiblichen.

\section{II}

Der Österreicher Michael Köhlmeier greift den Europa-Mythos dialektisch auf, als den „Gründungsmythos“, den der deutsche Bundespräsident Gauck in seiner Rede am 22. 2. 2013 im Schloss Bellevue als imaginäre Basis unserer europäischen Zukunft vermisst? ${ }^{7}$. Köhlmeier erzählt ihn neu, ausgehend von Bekanntem: Europa spielt

4. - J. Bobrowski, Europäische Ode, a.a.O., S. 193, V. 34-36.

5. - Gustav Schwab, Sagen des klassischen Altertums, Berlin, Insel Verlag, 2011 (Leipzig 1932), S. 30-36.

6. - Vgl. Almut-Barbara Renger, Nachwort, in Mythos Europa. Texte von Ovid bis Heiner Müller, hrsg. von A.-B. Renger, Leipzig, Reclam Verlag, 2003, S. 222-244, hier S. 244.

7. - https://www.bundespraesident.de/SharedDocs/Reden/DE/Joachim-Gauck/ Reden/2013/02/130222-Europa.html. 
mit dem gewaltigen, gewalttätigen Stier, packt ihn bei den Hörnern, verlässt ihr Vaterland und erprobt neue Lebensformen, ausgehend von einer Meerfahrt. Zeus zeugt mit Europa drei Söhne, der erste ist Minos, er wird König von Kreta, muss allerdings erfahren, dass seine Gattin einen von Poseidon geschaffenen Stier liebt, woraus der Minotauros entsteht. Diesen sperrt Minos in ein von Dädalos erbautes Labyrinth, in dem einem Ungeheuer Menschenopfer zugeführt werden. Zwei Liebesverhältnisse zwischen Frau und Stier, als Symbol sowohl ein Wasserwesen wie ein Himmelstier, führen zum Labyrinth, stiften bedrohliche, aber auch zu bewältigende Verwirrung8.

Köhlmeiers Deutung zu Beginn seines Sagenbuches soll ausführlich zitiert werden dürfen:

Das naive, hübsche Mädchen Europa wurde von Zeus, dem weißen Stier, durch das Meer nach Kreta getragen. Und dort am Strand verwandelte sich Zeus abermals, diesmal in einen Adler, er legte seine dunklen Schwingen über die kleine Europa und - und hier gehen die Meinungen auseinander: vergewaltigte sie, sagen die einen, liebte sie, sagen die anderen. Normalerweise spielt es keine so große Rolle, für welche Interpretation man sich entscheidet; in diesem Fall lege ich Wert darauf. Die anderen sagen: Nein, er vergewaltige sie nicht, es war eine Liebesbeziehung. Europa verliebte sich ihrerseits ebenfalls in ihren Entführer. Ich bin der Meinung, es ist schon entscheidend, ob unser Kontinent als Folge einer Vergewaltigung entstanden ist oder aus Liebe. Die Zyniker werden sagen: Schau dir doch die Geschichte Europas in den letzten paar tausend Jahren an, was soll das andres gewesen sei[n] als eine Vergewaltigung. Europa ist vergewaltigt worden, und Europa hat dann im Laufe seiner Geschichte die ganze Welt vergewaltigt. Das sagen die Zyniker. Ich bin kein Zyniker, und deshalb neige ich mehr zu der Liebesgeschichte. (S, 31)

Prüfen wir diese Deutung im Blick auf die Quellen. Nach Ovid ist sie „die Ent- oder Weggeführte“ (ablata, Ov. met. II 873), der der Stier die Furcht allmählich nahm, so dass sie sogar von sich aus den Ritt wagt, bis sie sich zu recht plötzlich auf dem offenen Meer ängstigt und zum verlassenen Strand zurückblickt. Von ihrem Ursprung her ist sie eine Reisende, Phönix' Tochter, aus dem Land der Phönizier, deren Kolonialisierung als Seevolk Griechenland entstehen ließ, als eine Kulturnation. Diesen „Kulturtransfer“ hat der Österreicher Raoul

8. - Michael Köhlmeier, Sagen des klassischen Altertums, München - Zürich, Piper Verlag, 2013 (1996), S. 23-30; Michael Köhlmeier, Das große Sagenbuch des klassischen Altertums, München - Zürich, Piper Verlag, 2012 [1999], S. 23-30, im Folgenden abgekürzt mit der Sigle „, dramatisch erzählt: http://ssl.br.de/fernsehen/br-alpha/sendungen/mythen/mythen-altertum-europa100.html. 
Schrott in seiner Neuübersetzung und -deutung der Göttergenealogie, der Theogonie, des Hesiod (2014) dargelegt. Europa stammt aus Asien, vom König Agenor aus der Stadt Tyros im heutigen Süd-Libanon (so in Herodots Historien, die „Tyrierin Europa“9), die sowohl auf einer Insel als auch auf dem Festland liegt. Europa verkörpert die Kultur der Phönizier als Vermittler zwischen Orient und Okzident, durch die Kulturleistungen des Alphabets, aber auch der Götterwelt, Basis der Lebensdeutung in Poesie und Philosophie. Europa ist selbst wie eine Seefahrerin dargestellt, sie hält - sitzend auf dem breiten Stierrücken, der sie wie ein Schiff aufnimmt - das Horn des Stieres mit der einen Hand (wie ein Steuer) und mit der anderen den Saum ihres purpurnen Kleides (Phönizien bedeutet „Purpurland“), sodass ,[h]och aufschwoll das weite Gewand der Europa/ Gleichwie ein Segel des Schiffes“ (Moschos) ${ }^{10}$.

Almut-Barbara Renger beschließt ihre Anthologie Mythos Europa mit Grünbeins Umdeutung der Europa-Gestalt: „Dass Europa umgekehrt ihr Schicksal in die eigene Hand nehmen könnte, war eine Pointe, mit der erst spätere Zeiten aufwarten sollten." ${ }^{11}$ Bobrowskis HandGestik ist Symbol der Moderne, da Gewalt durch Liebe versöhnt sein oder werden könnte. Der Weg durch das Labyrinth der Wege, Umwege und Irrwege vermag am selbst ausgelegten Faden gehändelt zu werden, glauben wir dies oder bleiben wir zynisch? Wie finden wir uns zurecht in Europa? Ist es nicht ein Labyrinth? Der Europa-Mythos ist schon in seinem minoischen Ursprung bestimmt vom „Erlebnis des Meeres“, da der „Stier als Wasserwesen“ und der Stierkult mit „Stieropfer und Stierspiele[n], an denen Frauen beteiligt waren", im chorischen Tanzen den Umgang mit dem unberechenbaren Element schulte ${ }^{12}$. Das Erlebnis des Meeres als Herausforderung soll im Folgenden im Blick auf Fahrende beiderlei Geschlechts ausgeweitet werden.

Neben Europa als erster weiblicher Mythengestalt Europas ist Ariadne zu nennen. Mit ihr beginnt Köhlmeier das letzte Kapitel seines Sagenbuches, es handelt vom Gott Dionysos, er gibt dem Kapitel den Titel Statt eines Nachworts, da diese Göttergestalt über die Antike hinausweist ${ }^{13}$. Ariadne folgt - wie Europa dem Stier Zeus - Theseus, für den sie ihren Vater Minos verraten hat, indem sie ihm durch den nach ihr benannten, berühmten Ariadnefaden einen Weg durch das

9. - Herodot, Historien, Buch IV, Kapitel 45, zit. n. Mythos Europa, a.a.O., S. 23.

10. - Moschos, Europa, Vers 1-36, 63-162, zit. n. ebd.,S. 25-30, hier S. 28f., nach Ov. met. II 875; vgl. Dürers in Venedig entstandene Federzeichnung von 1495.

11. - Mythos Europa, a.a.O., S. 221.

12. - Hans von Geisau, Europe, in Der kleine Pauly. Lexikon der Antike, München, Deutscher Taschenbuch Verlag, 1979, Bd. 2, Sp. 446-448, hier S. 447.

13. - Vgl. meine Dissertation „Des dunkeln Lichtes voll“. Hölderlins Mythokonzept Dionysos, München, Wilhelm Fink Verlag, 1987. 
Labyrinth gewiesen hat. Dabei sind das Problem - das Labyrinth und die Problemlösung - das Aufrollen des ausgelegten Fadens (filo relecto, Ov. met. VIII 173) - vom selben genialen Handwerker, Dädalos, erfunden bzw. verraten. Dies ist als durchkreuzter Blick auf den Geist des Erfinders und des Handwerkers festzuhalten, der gleichermaßen Labyrinth und Weg durch das Labyrinth erschafft. Sollte dies ein Signal und Symbol dafür sein, dass nach der Technik-Begeisterung die EthikErnüchterung folgt, nach dem Prometheischen das Epimethische, um einen anderen europäischen Mythos anzusprechen, mit Gilles Deleuze: „Ariadne ist es, die den Faden im Labyrinth festhält, den moralischen Faden"14? In jedem Fall handelt es sich bei den griechischen Mädchenmythen ,Europa und Zeus` wie Europas Enkelin ,Ariadne und Theseus“ wohl nicht um „Mädchentragödien“ im Sinne Walter Burkerts, sondern mythenstrukturell um Erfahrungen der Abreise von zu Hause sowie Perioden der Abgeschiedenheit und des Leidens, eben Odysseen.

Köhlmeier gelingt es, im Verlauf seines Sagenbuches in der Odyssee die philosophische Denkpotentialität und -aktualität unter der Erzählschicht von Seefahrer-Märchen und „Seemannsgarn“ (S, 172) aufzudecken. Er betont aber den durchgehenden FiktionalitätsCharakter der Ich-Erzählung am Hof der Phäaken, die das ästhetische Paradox der fiktiven Authentizität nicht auflöst. Übersetzungen der Odyssee sind Legion, jede Zeit findet ein ihr entsprechendes Modell: Auch Bobrowski hat sie 1955 in einer bis heute sehr erfolgreichen Neuausgabe Schwabs herausgegeben.

Odysseus als „,der heimkehrende Heimatlose“15 läuft willentlich oder unwillentlich eine Vielzahl von Inseln an. Darin erwarten ihn, „Stationen einer éducation sentimentale"16 in Begegnungen mit Frauengestalten. Mit einer Fülle von Weiblichkeitsrollen wird er konfrontiert, die ihn immer wieder von seiner Ehefrau Penelope ablenken: Kalypso, Kirke, die Sirenen. Vor allem aber die Begegnung der Königstochter Nausikaa mit dem wilden Mann Odysseus, dem nackten Schiffbrüchigen, erinnert an den Europa-Mythos. Wie Europa mit ihren Gefährtinnen die Blumenkörbchen mit Erotik symbolisierenden Blüten füllt, bereitet sich Nausikaa auf eine erotische Begegnung durch Waschen der Wäsche vor, gleichsam durch Weißen der Brautgewänder im Freien. Nach Köhlmeier (S, 171) verkörpert sie durch ihre Offenheit und Vorbehaltlosigkeit die Gastfreundschaft der Phäaken, die unerschrockene Haltung der Xenophilie als Kontrast zur Xenophobie. Dem Fremden wird Raum

14. - Gilles Deleuze, Kritik und Klinik, Frankfurt am Main, Suhrkamp Verlag, 2000, S. 136-114, zit. n. philosophie magazin, a.a.O., S. 90.

15. - Mythos Odysseus. Texte von Homer bis Günter Kunert, Hrsg. Bernhard Zimmermann, Leipzig, Reclam Verlag, 2004, S. 78.

16. - Ebd., S. 105. 
gegeben, er wird am Königshof der Phäaken integriert und aufgefordert, von sich zu erzählen. Diese Erzählkunst ist wie ein kompensatorisches Abenteuer unter undurchsichtigen Schicksals-Bedingungen, angesichts der Kontingenz des Lebens und Sterbens ein „Einwirken eines Fadens in den mythologischen Teppich“" (S, 270).

Dass Europa ein Ort des Erzählens sei, eines mutigen Sich-Äußerns vor Fremden über Eigenes, soll als philosophisch-ästhetische These mit Hannah Arendt begründet werden. Die Philosophin, aufgewachsen wie Kant in der Hafenstadt Königsberg am Sarmatischen Meer, gestaltet ihre Handlungstheorie unter dem Symbol des Fadens, der in einer Welt mit fadenscheinig gewordener Tradition erneut gefunden werden kann, muss und soll. Dabei wählt Arendt nautische Metaphern wie „Wind des Denkens“, der „,das Land des reinen Verstandes“ (nach Kant), ,eine Insel und durch die Natur selbst in unveränderlichen Grenzen eingeschlossen“, zugänglich macht. Diese Insel ist „umgeben von einem weiten und stürmischen Ozean“, den Tücken des Alltags und Lebens ${ }^{17}$. „The Broken Thread of Tradition“ (DT, 523) ${ }^{18}$ ist für Arendt Motivation ihres Werkes Vita activa, mit dieser Metapher beschreibt sie auch Martin Heideggers Wirkung als Lehrer der „Sache des Denkens“: „der, gerade weil ihm der Faden der Tradition gerissen ist, die Vergangenheit neu entdeckt“ $(\mathrm{M}, 183)^{19}$, , die totgeglaubten Bildungsschätze der Vergangenheit werden zum Sprechen gebracht" (M, 184). Für Arendt bildet sich die Polarität heraus: „Der Faden der Tradition und das Band der Menschheit“ (DT, 290), ist dies eine zeitliche Abfolge, eine historische Verpflichtung, eine Wandlung durch Anverwandlung, hier der Mythologie durch Neuerzählen, nach Arendt ein politischer Akt nach dem Modell des Phäakenhofes?

Nur in Klammern formuliert Arendt in einem Gedicht: ,,gedichtetes Wort/ ist Stätte, nicht Hort)“ (DT, 289). In der „Stätte“ ist ein Ort wichtiger Begebenheiten gegeben, feierlicher Handlungen mit besonderer Bedeutung für die Öffentlichkeit, sie ist nicht nur aufbe- bzw. verwahrend und museal abschließend wie ein Hort. Immer wieder tritt die Polis ins Blickfeld. In ihr entfaltet sich an Spiel-Stätten, wie dem Theater, die Ordnungskraft der Mythologie, d. h. ihre gedanklichdichterische Kraft, Ambivalenzen auszudrücken und die Zukunft unter Anknüpfung an die Vergangenheit zu gewinnen. Für Arendt ist die Polis mit Agora und Emporion der Ort und Raum der Öffentlichkeit, des

17. - Vgl. Hannah Arendt, Vom Leben des Geistes, München - Zürich, Piper Verlag, 2008 (1998), S. 192 und S. 207.

18. - Hannah Arendt, Denktagebuch, Zwei Bände, München - Zürich, Piper Verlag, 2003 (2002), im Folgenden mit der Sigle „DT“ abgekürzt.

19. - Hannah Arendt, Menschen in finsteren Zeiten, München, Piper Verlag, 2012, im Folgenden mit der Sigle „M“ abgekürzt. 
Erzählens. Sie betont die Bedeutung der Pluralität, in Form des Paares, schon von Adam und Eva her, als gleichzeitige Schöpfung eines „Wir“, jeder Form von Versammlung, die sich immer wieder neu konstituieren soll. In Arendts Begriff der Natalität liegt die Beschwörung des stets Neu-Anfangen-Könnens, nach dem mythologischen Muster Penelopes. Darin liegt Arendts Deutung Europas aus den griechischen Mythen nach Homers Narrativ: die Polis, eine als Ausgangspunkt gesetzte, immer neu sich generierende Gemeinschaftspraxis, von der sich die Bürger geistig herleiten (polites), nicht umgekehrt ein aus der Summe von einzelnen ,civis' errechnetes Abstraktum ,civitas', so Etienne Balibars Demokratie-Kritik mit Arendt ${ }^{20}$. Haben wir einen solchen Phäakenhof in Europa, an dem die Gründungs-Mythen erzählt werden können und woraus sich die politische Erzählgemeinschaft generiert?

\section{III}

In seinem Generationenroman Abendland ${ }^{21}$, einer Form der ,Anthropo-Gonie‘ des Zwanzigsten Jahrhunderts im Kontrast zu ,Theogonien' vergangener Zeiten, beschreibt Köhlmeier im Kapitel „Europa“ Begegnungen mit zwei Europäerinnen, deren Lebenswege intellektuell und ethisch-politisch die Dramatik europäischen Handelns ausloten und darin Hannah Arendt verschwistert sind: Edith Stein („Mitpatronin Europas“ seit Oktober 1999 als „eine Symbolfigur für Respekt und Toleranz in Europa“) und Emmy Noether. Der Terminus „Europa“ wird als „Okzident" und - auch etymologisch aus hebr. ereb = Dunkel, Abend - als "Abendland“ gedeutet, so dass der RomanTitel eine Weiterführung der Reflexion, ausgehend von Köhlmeiers Sagenbuch, darstellen soll.

Die Methode, zwei Figuren in einen Kontakt zu bringen, der zunächst willkürlich wirkt, nennt der Erzähler „,double-tale“ (A, 500), „Doppelporträts“ (A, 662), eine Erzählform, die von Plutarchs „Parallelbiographien“ hergeleitet werden kann. In Köhlmeiers Roman bezieht sich der Terminus direkt auf die Parallelisierung historischer und moderner Musikerbiographien, wobei über die antike Konstellation Orient-Okzident hinaus die Paarungen West- und Ost-Europa sowie Europa-Amerika befruchtend sind.

Beide Frauen, aus deutschsprachigem jüdischen Hause, gehören in eine Epoche Europas, in die erste Hälfte des Zwanzigsten Jahrhunderts (1882-1935, 1891-1942), und zeigen extreme Lebensmöglichkeiten;

20. - Etienne Balibar, Klassenkampf um die Demokratie? Zur historischen Dialektik von Demokratie und Bürgerschaft, polar Nr. 7, http://www.polar-zeitschrift. de/polar_07.php?id=315, <31.12.2014>.

21. - Michael Köhlmeier, Abendland, München, Hanser Verlag, 2007, im Folgenden mit der Sigle „A“ abgekürzt. 
sie stehen als Kontrastfiguren zu „Hitler and Stalin. Parallel Lives“22, Stein ist Opfer Hitlers, Noether hatte zunächst ins Exil nach Moskau gehen wollen, wäre dann wohl, wie ihr Bruder Fritz ${ }^{23}$, Opfer Stalins geworden. Damit schafft Köhlmeier ein Labyrinth von Assoziationen und Anspielungen, Parallelgeschichten sowohl im Guten als auch im Schlechten, sowohl der Opfer als auch der Täter, eine sehr durchwachsene Darstellung der durchkreuzten europäischen Geschichte des Zwanzigsten Jahrhunderts. Der Roman Abendland grenzt in diesen feinen Verästelungen an Unübersichtlichkeit und Undurchdringlichkeit; ein Erzählfaden ist - wenn überhaupt - nur mit äußerster Anstrengung zu konstruieren. Diese Lebensläufe sprengen Systematisierungen.

Die beiden jüdischen Frauen stellen sich ungeheuren Aufgaben, in der Philosophie bzw. Theologie und der Mathematik bzw. Physik, sie streben Habilitationen an, arbeiten dazu bei gegenüber der ,Frauenfrage, d. h. der ,Männlichkeitsfrage‘ im Reich der Wissenschaft, sehr ambivalenten Förderern namens Husserl und Hilbert, beide vergeblich, aber sie partizipieren an einer Wissenschaftler-Gemeinschaft, die europäisch arbeitet, durch Veranstaltungen mit Vortragenden ,aus dem weiten Europa“ (A, 206). Sie leben beide um den Ersten Weltkrieg in Göttingen, beide auf der Suche nach einem geistigen Prinzip trotz der und gegen die Kriegsleiden, schauen den Ungeheuern des Zwanzigsten Jahrhunderts in die Augen (Stein dem Nationalsozialismus, Noether dem Sündenfall der reinen Mathematik in der Atomphysik, im Kontakt zu Julius Robert Oppenheimer, der den Komplementaritätsgedanken der Quantenphysik als Möglichkeit eines doppelten Gesichtspunkts deutet, ,je nachdem, unter welchem Gesichtspunkt wir das Leben betrachteten, sowohl als Sterbliche als auch als Unsterbliche“, A, 263f.). Die Methode beider ist das Gespräch mit Jüngeren, hier mit dem Protagonisten Carl Jacob Candoris, für Köhlmeier ein Erzählfaden im Labyrinth des Abendlandes, das Europa heißt, eine Bildungsgeschichte über den mutigen Umgang mit Konflikten. Dieser Erzählfaden ist allerdings so komplex, dass er alle mythologischen Muster sprengt. Solche werden nur noch als scheinheilig zitiertes Bildungsgut von sich als Widerstandskämpfer stilisierenden Opportunisten verwendet, in dem ironisch zitierten Rechtfertigungsbuch: „Der Faden der Ariadne. Wie man in Irrsal und Wirrsal den richtigen Weg nicht verliert. Erinnerungen eines Physikers unter dem Nationalsozialismus" (von Wilhelm Jobst) (A, 628).

22. - Alan Bullock, Hitler and Stalin. Parallel Lives, London, HarperCollins, 1991. Für diesen Hinweis neben vielen anderen danke ich herzlich Michael Szczekalla.

23. - 1933 als Mathematikprofessor in Breslau entlassen, 1934 ins Exil nach Tomsk, Ende der Dreißiger Jahre verhaftet und zu 25 Jahren Gefängnisstrafe für Spionage verurteilt, September 1941 hingerichtet. 
Diese beiden Frauengestalten, Wissenschaftlerinnen, erscheinen zu einer Zeit, in der auf der Seite männlicher Dominanz der Krieg vorbereitet wird, unter dem Slogan während der Beerdigung von Edward VII. in London im Mai 1910: „Europa ist tot.“ (A, 104). Der RomanProtagonist, der seine Lebensbeichte vor seinem Biographen, dem Ich-Erzähler, ablegt, geboren 1906 in Meran, in einer Südtiroler Familie mit jüdischen Wurzeln (A, 89-93, 323f.), gestorben 2001, Professor der Mathematik an der Universität Innsbruck, nennt „Edith Stein, Emmy Noether" seine „Meister“ (A, 312). Beide Frauengestalten helfen ihm, dem Tod zu begegnen. Dieser Parallelismus wird vom Rezensenten Klaus Zeyringer ${ }^{24}$ auf eine Formel gebracht, die eine Schneise in den in einer Nacherzählung konstruiert und überfrachtet wirkenden Roman schlägt: „Die eine repräsentiert ein romantisches Deutschland, die andere ein aufgeklärtes - und in der deutschen Wirklichkeit endet die eine in Auschwitz, die andere im US-Exil.“ Die Wahlverwandtschaft der beiden Frauen, der außergewöhnlichen Menschen, realen Personen der Zeitgeschichte im Roman als Jahrhundertpanorama, wird immer wieder betont, aber was ist ihr geistiges Band, um sie zu wichtigen Begleiterinnen auf Candoris' „Odyssee zwischen Geist und Gewalt"25 zu machen? Verena Auffermann zeigt die Spannung zwischen den beiden Frauen auf:

Wenn Köhlmeier über Religion spricht, ohne die das Abendland ja kein Abendland wäre, dann tut er das nicht nach bekannter Manier österreichischer Hasstiraden, sondern vermittelt über Personen. Diskreter und zugleich direkter als über die tragische Lebensgeschichte Edith Steins kann man das Versagen der katholischen Kirche im Zweiten Weltkrieg kaum zur Sprache bringen.

Die weitestmögliche Entfernung vom religiös-mythischen ist die Mathematik und Physik. Und da Köhlmeier an Frauen mehr interessiert ist als an Männern und die vergangenen hundert Jahre ohne die Emanzipation der Frau nicht zu denken sind, lässt er Carl bei der Physikerin und Mathematikerin Emmy Noether studieren. ${ }^{26}$

24. - Klaus Zeyringer, „Gute Geschichten, denkwürdige Kreise. Michael Köhlmeiers großer Lebens- und Zeitroman umfasst das ganze ,Abendland', in all seinen Varianten, in vielen politischen Wechselbädern“, Der Standard, Wien, 18.8.2007, http://www.lyrikwelt.de/rezensionen/abendland-r.htm <22.12.2014>.

25. - Vgl. Harry Nutt, „Die Wahlverwandten“, Frankfurter Rundschau, 1.10.2007, http://www.fr-online.de/literatur/die-wahlverwandten,1472266,3134366. $\mathrm{html}<27.12 .2014>$.

26. - Verena Auffermann, „Das ganze Leben. Michael Köhlmeiers monumentales Jahrhundertepos ,Abendland“ wagt viel und gewinnt, Die Zeit, 20.9.2007, http://www. zeit.de/2007/39/L-Koehlmeier <27.12.2014>. 
Hier soll jedoch der gemeinsame Nenner herausgestellt werden, Europa als Mut zum Leben, als Neugier und unbändig freier Geist, neue Wege zu beschreiten, Neuland zu erfahren und dabei systematisch zu reflektieren, aber auch den Mythos der Liebe, verkörpert in der Beziehung zwischen Europa und dem Stier ${ }^{27}$, immer wieder neu zu erzählen. Die gemeinsame Methode beider Frauen ist das Gespräch, die Entwicklung von Gedanken in Vorträgen, ,in statu nascendi“ (A, 255), in Natalität und - bei stets vorausgesetzter Würdigung der Gesprächspartner - die Pluralität nach Hannah Arendt. Innerhalb der ganz in die Gegenwart gefügten Gespräche entstehen Figuren der geschlossenen Vollendung, im Erfassen des Unendlichen im Endlichen, sei es phänomenologisch-theologisch (Steins Arbeit Endliches und ewiges Sein. Versuch eines Aufstiegs zum Sinn des Seins, 1936) oder mathematisch-politisch, da Noether in der Kommutativen Algebra Revolutionäres leistete, indem sie auf Endlichkeit hinwies: „Hat jedes Ideal eines Ringes eine endliche Basis, so heißt der Ring noethersch.“28 Dabei gehen beide, die Phänomenologin wie die Mathematikerin, von den in Göttingen als Welthauptstadt gelehrten exakten Wissenschaften aus (A, 260) und kommen zu einem ,Nihilismus“, abstrahieren ihn, d. h. vom Tod bzw. von der Null, in der alles und nichts enthalten ist (A, 648). So fallen für Noether in der Mathematik ,Schein und Sein“ zusammen (A, 255), ,die Bausteine dieser ordnungserfüllten Welt [verhalten sich] so wild und unvorhersagbar" (A, 257), wie das Modell des Benzolrings von Kekulé als mythologische Ring-Schlange gesehen wird.

Edith Stein spricht mit Candoris und seinen exzentrischen Großtanten Franzi und Kundi Herzog (A, 71) - historisch als Frauenfiguren belegt in den Schwestern Anne und Pauline Reinach, die unter dem Tod des Philosophen und Husserl-Schülers Adolf Reinachs (A, 206) im November 1917 als Kriegsfreiwilliger leiden - über die Gefährdung durch SuizidGedanken (A, 204), innerhalb des Seligsprechungsprozesses wird geprüft, ob sie eine Heilige für Suizidgefährdete sein könne (A, 201); Emmy Noether befreit Candoris, ihren ehemaligen von Hilbert vermittelten (A, 254) Dissertator, vom Selbstvorwurf, ein Mörder zu sein.

Candoris trifft Stein als 8-Jähriger, also 1914 in Göttingen. Seit 1913 studiert Stein dort bei Husserl, 1916-18 als seine Assistentin, Candoris' Mutter ist gleichaltrig mit Stein (A, 72) und äußerst expressiv. Der Erste Weltkrieg wird von ihr als „Untergang Europas“ begrüßt (A, 212). Stein reflektiert in einem Brief an den 11-Jährigen im November 1917 (A, 245) schließlich den Umschlag der Kriegsbegeisterung in „Schmerz“, Suizidgedanken, Nihilismus, Stein stellt Fragen, die an die Deutung

27. - Vgl. Ruth K. Westheimer, Jerome Singeman, Mythen der Liebe, Gütersloh, Collection Rolf Heyne, 2010.

28. - Dtv-Atlas der Mathematik, Bd. 1, München, 2.A. 1976, S. 118. 
des Todes als „Reizimpuls für das Leben“ (A, 204) anknüpfen: „Der Tod als Kulturprodukt? Im Gegensatz zum Tod als einem natürlichen Ereignis?“ (A, 246) und antwortet: „Daß sie sich von dieser Welt abwenden wolle, daß sie in Kontemplation leben wolle. Wenn die Welt keinen Sinn brauche, brauche der Sinn keine Welt.“ (A, 248) Die von Stein in ihrer Dissertation (1916) entwickelte phänomenologische Methode wird erkennbar, eine erkenntnistheoretische, nicht psychologische „Einfühlung“ (A, 206) in kritischem Bewusstsein der Probleme einer egomanischen, usurpatorischen „Einsfühlung“, stattdessen Intersubjektivität, abstrakt möglich über die Zeiten hinweg, sowohl in die Vergangenheit als auch in die Zukunft.

Stein wird Patronin Europas genannt, ihr ,theoretischer Beitrag zu einem neuen Europa“ liegt einerseits in der erkenntnistheoretischethischen Methode der „Einfühlung“ als Schutz vor Vorurteilen in der phänomenologischen Urteilsenthaltung (epoché), als Akzeptanz des Andersseins (Alterität), und andererseits in ihrem Konzept einer philosophischen Anthropologie, nach der sich der Aufbau der menschlichen Person (1932/33) ${ }^{29}$ aus dem Verhältnis zwischen Individuum und Kultur, dem geistigen Erbe abendländischen Geistes ergibt, nach dem man gleichzeitig verschiedenen Gemeinschaften angehören kann, so z. B. als heutiger Mensch der überzeitlichen griechisch-römischen Kultur als Grundlage Europas. Stein ist in Leben und auch in ihrem staatspolitischen Werk Wegweiserin zu einer ,geschwisterlichen Gemeinschaft“ in Europa.

Emmy Noether wird in ihrem USA-Exil 1935 kurz vor ihrem Tod geschildert als ,,impulsiv, umweglos, kauzig und meistens warm“ (A, 301), aber ,politisch naiv“ (A, 621). Von dieser Vertreterin der Mathematik als einer „Nutzanwendung unnützer Erkenntnisse“ (A, 296) lernt Candoris, dass auch „Mathematik“, „Kunst der Tautologie“, der „Gleichung“, „elegantester Nihilismus“ sei (A, 648) und darin eine „Metapher für die Welt" (A, 649). Für Noether umfasst das „Mathematik-Reden“ auch Reflexionen über Ästhetik, die sich in Gesprächen über MythenTypisierungen wie, ,schöner Sieger vs. hässlicher Verlierer' entfalten (A, 271) und Noether - gegen einen ,,amoralischen Ästhetizismus“ (A, 255) bzw. ästhetischen Idealismus (A, 272) - zu Deduktionen über die „Dialektik der Schönheit“ (A, 292) inspirieren wie „Das Schöne ist eine Variable, das Häßliche eine Konstante“" (A, 284).

29. - Vgl. Angela Ales Bello, „Edith Steins theoretischer Beitrag zu einem neuen Europa“, in Europa und seine Anderen. Emmanuel Levinas, Edith Stein, Józef Tischner, hrsg. von Hanna-Barbara Gerl-Falkovitz, René Kaufmann und Hans Rainer Sepp, Dresden, Thelem Verlag, 2010, S. 19-32, hier S. 29-31. 


\section{IV}

Zweifelsohne scheint Köhlmeiers jüngster Roman Zwei Herren am Strand ${ }^{30}$ als Parallelbiographie in Gesprächen zwischen Winston Churchill (1874-1965) und Charlie Chaplin (1889-1977) dem Muster zu folgen, zwei Gestalten exemplarisch für eine entscheidende Phase der europäischen Geschichte ins Gespräch zu bringen, die Alliteration von „Edith“ und „Emmy“ spiegelt sich in der der beiden Herren. Allerdings ist dieser Roman wesentlich kürzer und durchsichtiger. Beide Ikonen ihrer Zeit verbinden sich im Kampf gegen den ,schwarzen Hund“, einerseits ihre Depression, andererseits Hitler. Beide Engländer, ja Londoner, sind mehrfach künstlerisch begabt, stilisierten sich zu einer Marke. Beide treffen sich zufällig - ohne sich gegenseitig vorzustellen - am Rande einer Party 1927 in Santa Monica (USA) zu einem Spaziergang am Pazifik (Z, 27), um über ihr persönlichstes Thema, den Freitod, zu sprechen. Daraus entstehen lebenslange regelmäßige Gespräche als „,talk-walks“ oder „,duck-walk-talks“ (Z, 20), „Doppelgänger“ trotz unterschiedlicher Physiognomie, Herkunft und politischer Ansichten (Z, 27f.), ihre Leidenschaft ist „Selbstmordarten zu sammeln“, aber auch Schutzmethoden: Churchill malt Landschaften im ewig unvollendeten Versuch, die Farbe des Himmels, sein Blau, zu treffen (Z, 56f.), Chaplin schreibt mit der "Methode des Clowns“ einen Brief an sich selbst, nackt liegend auf einem weißen Blatt kreisend im Strudel der Worte, dem „Mahlstrom“ (Z, 59f.). Damit gewinnen Churchill und Chaplin zwei Methoden, ihren Weg im Labyrinth ihrer Zeit und ihrer Persönlichkeit, in Außen- und Innenwelt zu finden, sie überleben in äußeren wie inneren Kämpfen und werden noch berühmter, im Kontrast zu den äußeren Schicksalen Steins und Noethers.

Sollte von den leichter zugänglichen Gestaltungen der männlichen Romanfiguren des Jahres 2014 ein Weg eröffnet sein für die immer noch ausstehende Würdigung der Lebensleistung der beiden Frauen als Europäerinnen, wie sie Köhlmeier hochambitioniert im Roman des Jahres 2007 vorstellt: zu den Kreisbewegungen in zwei weniger zugänglichen, labyrinthisch komplexen Werken rezeptionsgeschichtlich vorzustoßen, zu der Phänomenologin und Pädagogin, die das Unendliche im Endlichen „einfühlen“, die Farbe des Himmels fassen will, zu der Mathematikerin und Physikerin, nach deren Theorem gilt: $\mathrm{Zu}$ jeder kontinuierlichen Symmetrie (Invarianz) eines physikalischen Systems gehört eine Erhaltungsgröße (Konstanz), eine von ihr bewiesene wechselseitige Bedingtheit von Invarianz und Konstanz, die bis heute wissenschaftsgeschichtlich gerühmt wird?

30. - Michael Köhlmeier, Zwei Herren am Strand. Roman, München, Hanser Verlag, 2014, im Folgenden abgekürzt durch die Sigle „Z“. 
Hat es Gründe, dass Köhlmeier als Österreicher - wie auch Robert Menasse $^{31}$ - anders auf die Europa-Idee blickt als deutsche Autoren, bei denen der Dominanz-Verdacht des Deutschen gilt? Vermag Köhlmeier durch die Erweiterung der Frage auf die Spannung zwischen Abendland und Amerika hier den Blick zu öffnen auf eine erweiterte Verantwortung Europas und Amerikas, ihre Werte gegenüber dem Orient bzw. dem Osten, sei es Moskau, sei es Polen, zu reflektieren? Inwieweit ist die jüdische Frage als eine europäische Frage des Zwanzigsten Jahrhunderts die entscheidende? Was wurde hier, durchkreuzt ${ }^{\star}$, die Linie des jüdischchristlichen Humanismus, das Vergessen und Verleugnen einer geborenen Geschwisterschaft und einer andauernden Verschwisterung? Steins Wunsch, Judentum und Christentum zu vereinen, war ihrer Zeit weit voraus.

Europa wird im Zwanzifsten Jahrhundert durchkreuzt von Kriegen und der Katastrophe der Shoah. Nur in der Erzählung als Erinnerung an dieses Kreuz kann Leben im Geschichtsbewusstsein möglich sein. Allerdings ist die Aufzeichnung über die Lebenswege so komplex, dass ein Faden im Labyrinth nicht gefunden werden kann, wenn, dann nur im endlos weiterzuführenden Erzählen und Zuhören. Candoris äußert sich am Lebensende als, ,frommer Atheist' (A, 108) mit gleichsam von Stein geliehener Stimme: „Lieber Gott, zeig mir den Weg, ich will ihn gehen!“ (A, 55), die „via regis“ als „via crucis“? Welche Stimmen leihen wir uns, welche sprechen aus uns, in phänomenologischen oder mathematischen Gottesbeweisen (wie bei Kurt Gödel, A, 59 und 299) oder besser Erzähl-Erweisen der Unabschließbarkeit der Reflexion? Zeigen solche Geschichten wie die Steins und Noethers, dass „der Glaube an die Vernunft" „die wesentliche Grundlage der zivilisierten Menschheit" ist (A, 56), dass dieser aber auch durchkreuzt werden kann durch eine andere Schönheit, die sowohl Logik als auch Erotik überwindet (A, 58), die Schönheit der Kunst, die die Hässlichkeit der Geschichte integriert und nicht verschweigt? Bei den beiden Frauen sucht Candoris „Genie[s] und Heilige des Trostes“ (A, 332); Trost geben dem Erzähler Lebensgeschichten, in denen ,fiction statt facts“ (A, 339) als Wahrheitsapproximation gewählt werden. Es sind immer mindestens zwei Erzählstränge gleichzeitig möglich. Aber beide Frauengestalten belegen, dass nur der „Ariadnefaden des gesunden Menschenverstandes [...] sicher durch das Labyrinth" der eigenen Erlebnisse und wissenschaftlichen Ergebnisse führt ${ }^{32}$.

31. - Vgl. Robert Menasse, Der europäische Landbote, München, Paul Zsolnay Verlag, 2012.

32. - Hannah Arendt, Zwischen Vergangenheit und Zukunft, München - Zürich, Piper Verlag, 2012 (1994), S. 114. 
Wie bezwang und bezwingt Europa den Stier? Vielleicht nach dem „Perseus-Komplex“ (A, 339), den Köhlmeier als Idee einer seiner Figuren zitiert: Wähle nicht den direkten Blick ins Auge des Tieres, sondern den Blick in die Ferne, in die Zukunft, ins lebendig Offene. So ist es angebracht, dass Köhlmeier den 8. Europäischen Mediengipfel in Lech unter dem Motto „Wer regiert Europa? Politik zwischen Ohnmacht und Gestaltungskraft" am 4.12.2014 eröffnet hat, mit pessimistisch-provozierender Forderung nach mehr Partizipation, denn das Volk sei - als politikverdrossene Übermacht der Passivität - der einzige Feind der Demokratie. Wie verändert sich die Zukunft, wenn politische Strukturen und Medien „fadenscheinig“ werden? Der indirekte Blick der Kunst ist allerdings - wie in Köhlmeiers Europa-Epos Abendland erwiesen - kein „Schönreden“ (A, 348), er ist aber - nach Arendt „,vita activa“ als „,amor mundi“, für ein Abendland, nach Bobrowski ein Schattenland ${ }^{33}$ als vergangenes, ein Traumland als zukünftiges, ein Vaterland als gegenwärtiges. und Stalin, München, C. H. Beck Verlag, 2.A. 2011. 
